\title{
Nonsorted circles - ein Vergleich zwischen arktischen und alpinen Formen
}

\section{Nomenklatur und Morphographie}

In der Literatur sind für die in dieser Arbeit untersuchten Formen verschiedene Termini zu finden, die alle mehr oder weniger als Synonyme aufzufassen sind:

GRIPP (1927) spricht von «Brodelstellen», WASHBURN $(1956,1969,1973)$ verwendet den Begriff «nonsorted circle». In LUNDQVIST (1962) wird die gleiche Form entweder als «nonsorted circle» oder als «mud circle» bezeichnet. HOPKINS und SIGAFOOS $(1951,1954)$ verwenden die Begriffe «frost scars», "peat rings» und «tussock rings». Außer diesen gebräuchlichsten Begriffen werden in der Literatur eine Reihe weiterer, nicht allgemein geläufiger Ausdrücke verwendet (z. B. «Gährlehmbeulen», "spot médaillons», «mud pits»). Keiner dieser Begriffe wird der Form ganz gerecht (deshalb wohl auch diese Vielzahl von Bezeichnungen), doch der in dieser Arbeit verwendete Ausdruck «nonsorted circle» beschreibt immerhin ein ganz wesentliches Merkmal dieser Erscheinungen (vgl. unten).

Bei den nonsorted circles handelt es sich um Kahlstellen in einer sonst gänzlich geschlossenen Vegetationsdecke. Ihr Vorkommen beschränkt sich auf die Tundrengebiete der höheren Breiten sowie auf die Hochgebirgsrasenstufe gemäßigter und niederer (GRAF, 1971) Breiten. Diese Kahlstellen weisen auf horizontalen Flächen eine runde bis ovale (manchmal auch eine sehr unregelmäßige) Form mit einem Durchmesser von etwa 0,3 bis 2 Meter auf. Am Hang ist ein Übergang in langgezogene, dem Gefälle folgende Formen zu beobachten. Häufig sind die nonsorted circles sekundär gegliedert, in Spitzbergen durch Zellenböden und auf dem Col de l'Iséran hauptsächlich durch deutlich ausgebildete Steinpolygone von 5 bis $20 \mathrm{~cm}$ Durchmesser, also durch Strukturböden. Damit ist schon gesagt, daß eine Sortierung nicht auszuschließen ist, wie es der Name vermuten lassen könnte. Entscheidend ist aber, daß die ganze primäre Form, also der eigentliche nonsorted circle, nicht durch eine Materialsortierung entstanden ist wie beispielsweise die Steinringe. Trotzdem zeigen einzelne Vertreter dieser Formen einen gewissen Zusammenhang mit den Strukturböden, weil nämlich die größten Steine auch hier in den randlichen Partien anzutreffen sind (Abb. 1 und 5).
Es sind vielleicht weniger die äußerlich sichtbaren morphologischen Merkmale der Form an sich, die eine separate Stellung rechtfertigen, als vielmehr das Zusammenspiel von Kahlstelle und Vegetation, das sich - wie weiter unten noch ausgeführt wird - auch im Untergrund manifestieren kann.

\section{Verbreitung der nonsorted circles}

Die zonale Verbreitung in den Tundrengebieten und der Hochgebirgsrasenstufe zeigt, daß es sich dabei um Regionen handelt, in denen eine intensive Wirkung des Bodenfrostes mit Sicherheit anzunehmen ist (oft sogar in Zusammenhang mit Permafrost). Die Obergrenze des Verbreitungsgebietes ist sicher dort zu suchen, wo die Mattenstufe in die Frostschuttstufe übergeht, bzw. dort, wo sich nonsorted circles wegen mangelnder oder fehlender Vegetation nicht mehr bilden können. Dieser Obergrenze im Hochgebirge entspricht eine polare Grenze im Tundragebiet. Die untere Begrenzung im Hochgebirge (bzw. die gegen Süden weisende Begrenzung im Tundragebiet) ist weniger eindeutig, sie ist aber dort anzusetzen, wo die Vegetation so kräftig wird (im Zusammenhang mit der nachlassenden Wirkung des Frostes), daß eine in ihr einmal geschaffene Verletzung wieder überwachsen werden kann.

Die bis heute eher spärlichen Hinweise auf nonsorted circles, die doch zonal eine ausgeprägte Verbreitung erwarten lassen, sind auf zwei Ursachen zurückzuführen:

1. Die für die Formbildung optimalen Verhältnisse werden nur selten erreicht, weil eine Anzahl von Faktoren die Genese beeinflußt. Als Beispiel sei der Faktor Schnee erwähnt, der die Bildung eines nonsorted circles absolut verhindern kann, wenn er in zu großer Mächtigkeit (Isolation!) während einer Zeit liegt, in der ein starkes Eindringen des Frostes in den Boden zu erwarten wäre. Gerade die Kuppenlage der nonsorted circles (Abb. 3) auf dem Col de l'Iséran deutet auf eine relativ geringmächtige Schneedecke in den kalten Monaten hin. Dafür sprechen auch die Vegetationsverhältnisse an dieser Lokalität, bei denen es sich um ein

Dr. Peter Fitze, Geographisches Institut der Universität Zürich, Blümlisalpstraße 10, 8006 Zürich 
Ineinandergreifen von Nacktried- und Krummseggenrasen handelt. Nacktriedrasen in reiner Ausbildung muß als extrem frosthart taxiert werden (mdl. Mitt. Dr. H. Hartmann).

2. Die nonsorted circles sind tatsächlich zu wenig genau erfaßt worden. Gerade wegen ihrer nicht immer sehr eindeutigen morphologischen Ausprägung werden sie vermutlich bald zu den Formen der gebundenen Solifluktion (beispielsweise zur Kategorie der Girlanden) und bald zu den Strukturböden gezählt.

\section{Hinweise zur Morphogenese aufgrund der Literatur}

Nach FROEDIN's Meinung (1918, in LUNDQVIST 1962) dringt der Frost an Stellen mit dünnerer oder sogar fehlender Vegetationsdecke früher und tiefer in den Boden ein als unter dichter Vegetation, was zu einem lokalen Aufbeulen beim Gefrieren führt. Damit ist wohl die Bildung und die Vergrößerung solcher Kahlstellen sowie auch die manchmal zu beobachtende Sortierung erklärt, nicht aber Aufpressungen von Material aus dem Untergrund an die Oberfläche oder seitliche Materialverlagerungen im Untergrund, wie sie verschiedentlich beobachtet worden sind (HOPKINS und SIGAFOOS 1951, SEMMEL 1969, WASHBURN 1973, eigene Untersuchungen in dieser Arbeit). Im Unterschied zur Deutung FROEDIN's handelt es sich dabei um partielle Materialverkagerungen, die einer andern Erklärung bedürfen. So können die Ursachen beispielsweise bei den recht komplizierten und noch wenig untersuchten Vorgängen beim Vorrücken der Frostfront in den Boden zu suchen sein. Auch der kryostatische Druck (WASHBURN 1956, p. 842) kommt bei gewissen Lokalitäten als mögliche Ursache partieller Materialverlagerungen in Frage. Unter diesem Begriff wird der Druck verstanden, der sich beim Eindringen einer Frostfront in den Boden bildet, weil ungefrorenes Material zwischen dieser und einer Permafrostoberfläche immer mehr eingeengt wird. Durch eine fortschreitende Erhöhung dieses Druckes kommt es schließlich an einer Schwächestelle zum Durchbruch (vertikal oder lateral). Währenddem HOPKINS und SIGAFoos (1951) Permafrost als unbedingte Voraussetzung dafür ansehen, ist schon verschiedentlich darauf hingewiesen worden, daß Permafrost beispielsweise durch ein dichtes anstehendes Gestein ersetzt werden kann (z. B. WASHBURN 1969, p. 91).

HOPKINS und SIGAFOOS $(1951,1954)$ haben in Alaska nonsorted circles genauer untersucht und sind dabei zu folgenden Schlüssen gekommen:

In einer anfangs geschlossenen Vegetationsdecke entstehen aus verschiedenen Ursachen (Beweidung, Zerreißen beim Gefriervorgang, starke Frosteinwirkung an schneefrei geblasenen Stellen, ursprüngliche Materialinhomogenitäten) Risse und Kahlstellen. Hier kann nun der Frost bevorzugt in die Tiefe wirken, da die unverletzte Vegetationsdecke daneben ja meist als guter Isolator wirkt. Im Untergrund dieser Kahlstellen ist also im Herbst ein rasches Eindringen des Frostes zu erwarten. Durch die Ausdehnung des feuchten Bodens beim Gefrieren resultiert einmal eine Aufbeulung der Kahlstelle im Sinne von FroEdIN. Die gefrorene Deckschicht wird nun durch das Vorrücken der Frostfront immer mächtiger und der kryostatische Druck im Untergrund nimmt zu. Es kann auf diese Weise noch nicht gefrorenes Material zur Seite gepreßt werden, wo unter der Vegetation der Frost noch nicht oder jedenfalls noch nicht so tief in den Boden eingedrungen ist. Dieses seitliche Herauspressen ist häufig mit einer Gegenbewegung verbunden, so daß Material von der Seite her in den Unterboden des nonsorted circles eindringen kann.

Es dürfte allerdings sehr schwierig festzustellen sein, ob dieser Vorgang tatsächlich genau so abläuft (Messungen während des ganzen Gefriervorgangs existieren bis heute nicht). Wichtig ist aber, daß das seitwärts verlagerte Material nachträglich eindeutig nachgewiesen werden kann, und daß es offenbar möglich ist, mit Hilfe des kryostatischen Druckes und den Vegetationsunterschieden an der Oberfläche solche Bildungen zu erklären.

Eine Bemerkung der beiden Autoren (1951, p. 97) ist im Zusammenhang mit der Genese noch hinzuzufügen: Es wird erwähnt, daß in steinigem Material eine Sortierung stattfindet und anstelle unsortierter Formen Steinpolygone gebildet werden. Danach wäre eine Unterteilung in sortierte und nichtsortierte Formen rein materialbedingt. Etwas anders sieht allerdings SEMMEL $(1969$, p. 59) den Unterschied zwischen diesen beiden Merkmalsgruppen, wenn er davon spricht, da 3 die «nonsorted circles und nonsorted stripes» der Moos-Frostschuttzone in den Kerngebieten der Frostschuttzone von sortierten Formen abgelöst werden. 
Abb. 1 Nonsorted circle, Westspitzbergen (Pickellänge $50 \mathrm{~cm}$ ). Diese Form ist aufgegraben in Fig. 1 dargestellt. Zu beachten ist die sekundäre Gliederung durch einen Zellenboden sowie die größeren Steine, die sich am Rand der Form befinden und deutlich kantengestellt sind.

Abb. 2 Nonsorted circle, Col de l'Iséran (Spatenlänge $65 \mathrm{~cm}$ ). Deutlich ist das sekundär entstandene Steinnetzwerk zu erkennen.

Abb. 3 Kuppe auf 2800 m Höhe am Col de l'Iséran. In der Vegetation befinden sich zahlreiche Kahlstellen (z. B. beim Spaten) von unterschiedlicher Form und Größe.
Abb. 4 Langgestreckter nonsorted circle, Col de l'Iséran (Spatenlänge $50 \mathrm{~cm}$ ). Diese hangaufwärts fotografierte Form ist undeutlich sekundär gegliedert.

Abb. 5 Nonsorted circle, Westspitzbergen (Länge der Rechteckseiten 60 und $40 \mathrm{~cm}$ ). Lage des Schnittes, von Abb. 6 durch schwarze Linie markiert.

Abb 6 Beispiel eines Schnittbildes des in Abb. 5 untersuchten nonsorted circles. Die Mächtigkeit der relativ skelettarmen Schicht, in der sich unter der Kahlstelle die deutlich steilgestellten Blöcke befinden, beträgt ungefähr $35 \mathrm{~cm}$.
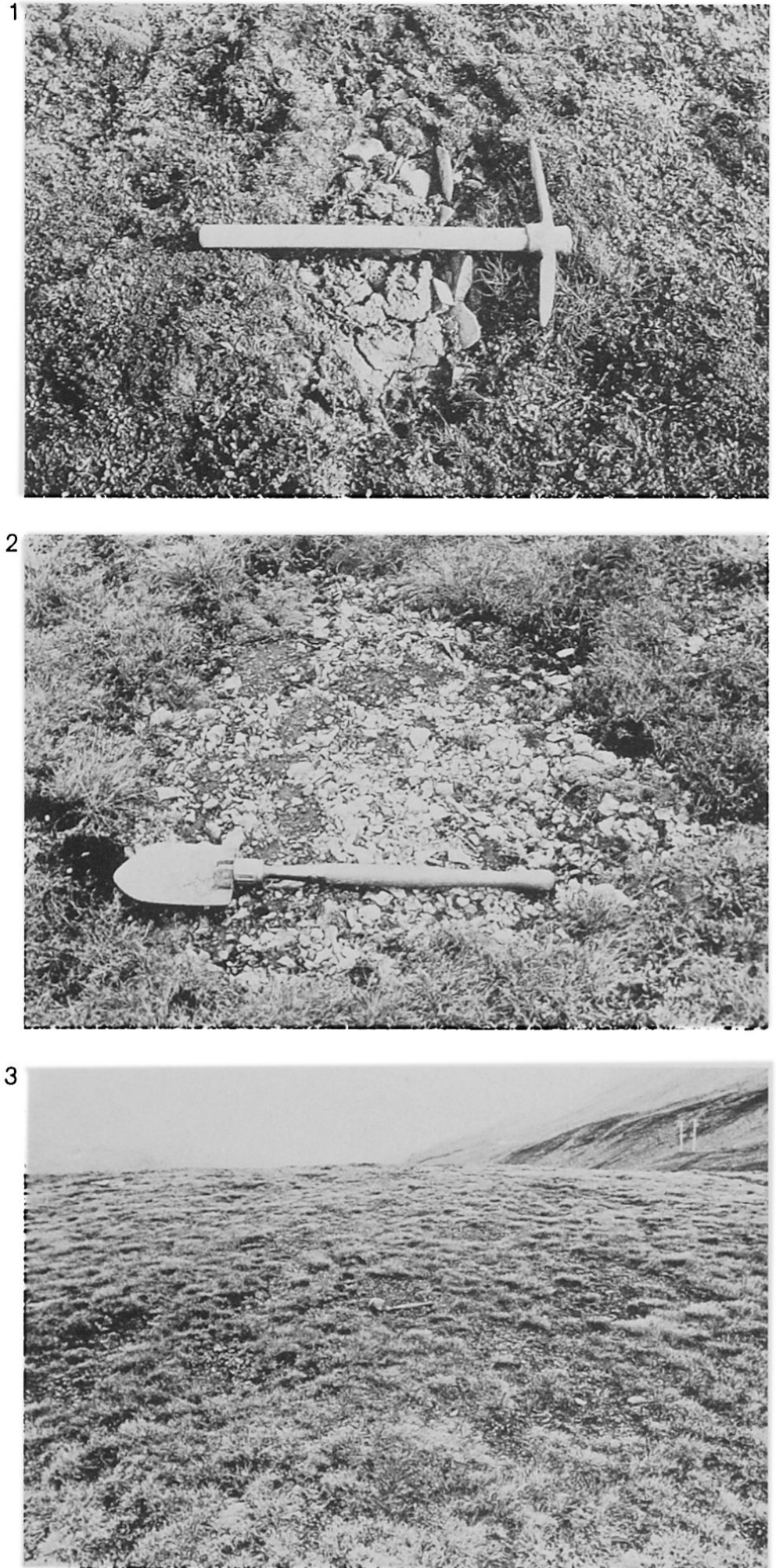
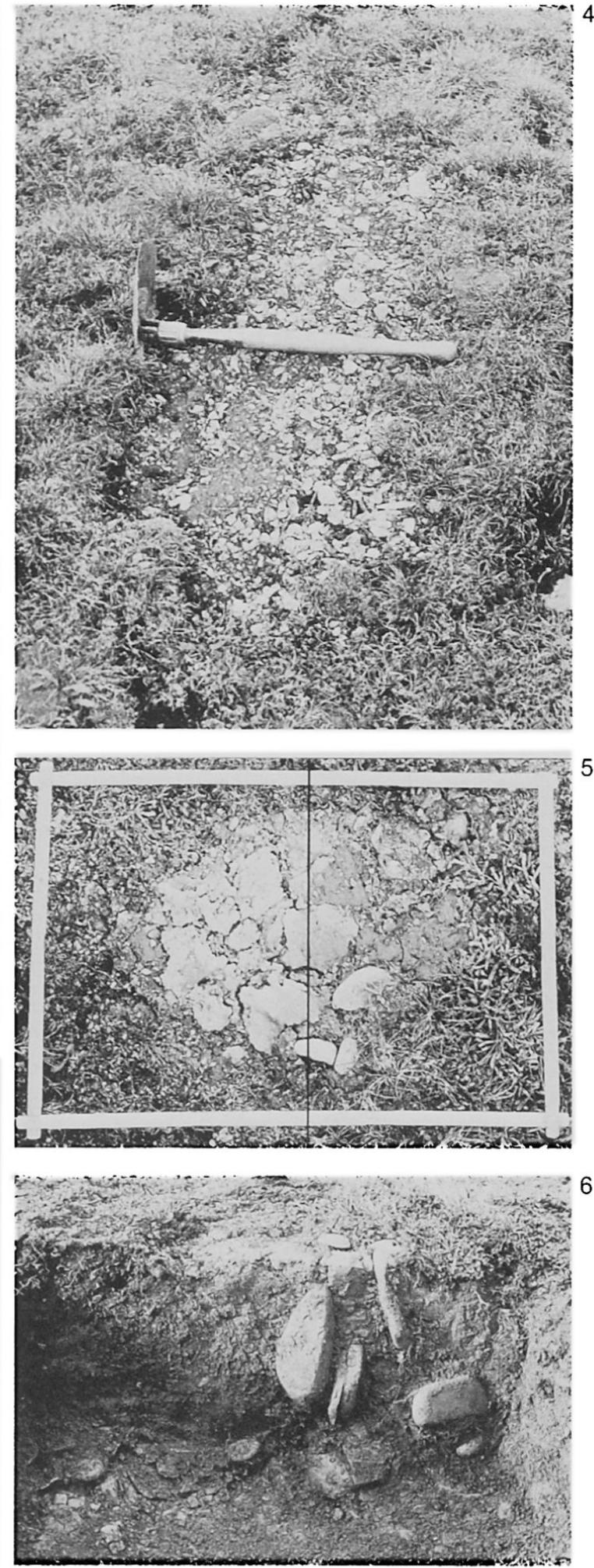


\section{Eigene Beobachtungen und Untersuchungen}

\section{Westspitzbergen}

Im Sommer 1970 wurden einige Beobachtungen an nonsorted circles etwa 5 Kilometer nordwestlich von Longyearbyen am Adventfjorden gemacht. Die Beobachtungsstelle liegt 30 Meter über dem Meeresspiegel, der Untergrund wird von stark verwitterten kretazischen Sand- und Siltsteinen gebildet (vgl. dazu MAJOR und NAGY, 1972). Alle untersuchten nonsorted circles sind in einer dichten Dryas-Tundra, im sog. Tetragono-Dryadetum (mdl. Mitt. Dr. H. Hartmann) zu finden (Abb. 1, 5, 6). Die Permafrostoberfläche wurde bei keiner der aufgegrabenen Formen erreicht, sie ist aber mit Hilfe des Bohrstockes in etwa 60 bis $80 \mathrm{~cm}$ Tiefe nachgewiesen worden.

Alle drei aufgegrabenen Beispiele zeigen an der Oberfläche ein ähnliches Bild: Eine absolut vegetationsfreie ovale Stelle von 30 bis $50 \mathrm{~cm}$ Durchmesser ist leicht eingesenkt in die dichte Tundra. Alle Kahlstellen sind sekundär durch Zellenböden gegliedert.

Der erste aufgegrabene nonsorted circle (Abb. 1, Fig. 1) zeigt unter der Kahlstelle eine feinmaterialreiche kesselartige Form. Ob dieses skelettarme Material als Ergebnis einer Sortierung betrachtet werden kann, ist ungewiß, denn es kann sich ja auch um eine natürliche Inhomogenität im Boden handeln. Erstaunlich ist bei allen 4 entnommenen Proben der durchwegs hohe Gehalt an organischem Kohlenstoff, am auffälligsten bei den Proben 1 und 2 (in Fig. 1), über denen ja keine organische Auflage und kein $\mathrm{Ah}_{\mathrm{h}}$-Horizont nachzuweisen ist. Auch die rezente Durchwurzelung kann für diese hohen Werte nicht verantwortlich gemacht werden. Dieselbe Beobachtung wurde auch bei der zweiten Aufgrabung (Fig. 2) gemacht. Eine exakte Erklärung für dieses Phänomen kann in dieser Arbeit nicht gemacht werden, denn die Aussagekraft dieser wenigen Proben ist gering. Es könnte sich dabei um mit Wasser in die Tiefe verlagerte humose Substanzen oder aber um intensive Durchmischungsvorgänge von humosem Material mit dem Mineralboden handeln. Als Vergleich sei überdies hinzugefügt, daß unsere mitteleuropäischen Braun- und Parabraunerden kaum höhere Werte im Ah-Horizont zeigen! Von der Korngröße her sind fast keine Unterschiede festzustellen, es handelt sich bei allen Proben um schluffig-lehmiges Material. Die beiden Proben 1 und 2 sind in der
Zusammensetzung der Feinerde praktisch identisch. Das kann wieder zufällig sein oder aber es besteht tatsächlich ein Zusammenhang zwischen diesen beiden Proben im Sinne einer vertikalen Materialverlagerung.

Der zweite aufgegrabene nonsorted circle (Fig. 2) zeigt 3 dunkle «verwürgte» Horizonte in einer Tiefe zwischen 5 und $25 \mathrm{~cm}$ unter der Kahlstelle. Die Laboruntersuchungen zeigen eindeutig, $\mathrm{da} B$ es sich dabei um Teile von humosen Horizonten handeln muß, mit vereinzelt extrem hohen Werten (maximal 5,8\% organischer Kohlenstoff). Auch das übrige Material weist die schon erwähnten hohen Werte an organischem Kohlenstoff auf. Es stellt sich somit die Frage nach der Herkunft dieser humosen Horizonte. Ein fossiler Boden konnte nirgends gefunden werden, so daß die Annahme wohl berechtigt ist, daß es sich um Teile des rezenten $\mathrm{A} h$-Horizontes handelt, die von der Seite her eingepreßt worden sind. Vielleicht würde sich bei genaueren Untersuchungen sogar eine Verbindung des rezenten $\mathrm{A}_{h}$-Horizontes mit dem eingepreßten Horizont ganz rechts (über den mittleren «Fortsatz») herstellen lassen. Auch die Werte im linken Teil von Fig. 2 zeigen keine eindeutige Trennung zwischen dem rezenten und dem eingepreßten humosen Horizont. Diese Hinweise zeigen deutlich, daß einige Anzeichen für eine seitliche partielle Materialverlagerung sprechen.

Die dritte Grabung schließlich hatte zum Ziel, die räumliche Verteilung der größeren Steine im Untergrund $\mathrm{zu}$ erfassen, um eventuelle Indizien für das Ausfrieren von Steinen zu erhalten, wie sie für Sortierungsvorgänge typisch sind. Schon bei der ersten Grabung hat sich ja gezeigt, da $B$ im Untergrund eine Art Feinerdekessel vorhanden (Fig. 1) und an der Oberfläche insofern eine Sortierung feststellbar ist, als sich alle größeren Steine im Randbereich des nonsorted circles finden (Abb.1). Zudem ist auch die Kantenstellung dieser Steine eindeutig. Gerade diese beiden Merkmale, Anhäufung im Randbereich und Kantenstellung gelten als typische Merkmale von Strukturböden. Um Ausfrierprozesse erfassen zu können, bot sich mir folgender Weg an:

Ein nonsorted circle (Abb. 5) wurde schrittweise durch eine Serie von parallelen Vertikalschnitten (in Abständen von $5 \mathrm{~cm}$ ) geöffnet. Die Aufnahme jedes Schnittes erfolgte mit Hilfe eines fest montierten Fotoapparates. Auf diese Weise konnte die Verteilung Grobskelett- 
Feinerde in insgesamt 15 Bildern festgehalten werden. Ein Beispiel eines solchen Vertikalschnittes gibt Abb. 6 wieder. Aus allen Schnitten geht klar hervor, daß eine 40 bis $60 \mathrm{~cm}$ mächtige skelettarme Schicht über einer sehr grobskelettreichen verwitterten Sandsteinschicht liegt (vgl. Abb. 6). Auf jedem Bild konnte mit einem Raster die Gesamtfläche der skelettarmen Schicht sowie der prozentuale Flächenanteil, der auf Steine mit einem Durchmesser von mehr als $2 \mathrm{~cm}$ entfiel, ermittelt werden. Der gemittelte Prozentwert aus allen Bildern ist angenähert proportional dem tatsächlichen Volumen im ganzen Bodenkörper. Innerhalb der skelettarmen Schicht wurden nun 2 Zonen unterschieden: Eine Zone senkrecht unter der Kahlstelle und eine zweite Zone bestehend aus den beiden Säulen unter der Vegetation. Die nachstehende Zusammenstellung zeigt klar, daß der volumenmäßige Prozentanteil der Steine in diesen beiden Zonen ganz unterschiedlich ist:

\begin{tabular}{lll} 
Grobskelettanteil & $\begin{array}{l}\text { Unter } \\
\text { Kahlstelle }\end{array}$ & $\begin{array}{l}\text { Unter } \\
\text { Vegetation }\end{array}$ \\
$\begin{array}{l}\text { in Volumenprozenten } \\
\begin{array}{l}\text { Feinmaterialanteil } \\
\text { in Volumenprozenten }\end{array}\end{array}$ & 13,5 & 7,3 \\
\hline
\end{tabular}

Unter der Kahlstelle liegen also volumenmäßig fast doppelt so viel Steine mit einem Durchmesser von mehr als $2 \mathrm{~cm}$ als unter der Vegetation. Anders ausgedrückt - falls es sich dabei nicht um einen Einzelfall handelt - kann daraus geschlossen werden, daß sich die größeren Steine bevorzugt in der Bodensäule unter der Kahlstelle befinden. Die eindrückliche Senkrechtstellung (vgl. Abb.6) unter der Kahlstelle ist im Zusammenhang mit dieser Beobachtung ein wichtiges Indiz für einen Ausfrierprozeß, ähnlich wie bei der Strukturbodenbildung. Auch der vertikal gestellte mittlere Humushorizont in Fig. 2 deutet in die gleiche Richtung.

Zusammenfassend lassen sich die Beobachtungen, die in Westspitzbergen an nonsorted circles gemacht wurden - immer unter dem Vorbehalt des sehr geringen Materials - wie folgt deuten:

- Hebungsvorgänge verbunden mit partieller vertikaler Materialverlagerung können durch verschiedene Beobachtungen belegt werden.

- Hinweise auf Sortierungsvorgänge und damit einen genetischen Zusammenhang mit Strukturböden sind nicht zu übersehen.
- Seitliche Materialverlagerungen im Untergrund sind mit großer Sicherheit anzunehmen.

\section{Col de l'Iséran}

Dieser $2800 \mathrm{~m}$ hohe Paßübergang verbindet die Ortschaft Val d'Isère mit dem Tal des Arc. Geologisch wird der Col durch die Piemontesischen Bündnerschiefer gebildet (vgl. DEBELMAS 1970). Die untersuchten nonsorted circles befinden sich auf einer kleinen Kuppe direkt neben der Paßhöhe (Abb. 3). Im Gegensatz zu Westspitzbergen hinterlassen diese Formen einen etwas uneinheitlichen Eindruck: Sie sind häufig girlandenähnlich ausgebildet, oft langgestreckt hangabwärtsgerichtet (Abb.4), manchmal aber auch isohypsenparallel. Vor allem ihre Ausmaße sind im Durchschnitt wesentlich größer als jene Westspitzbergens. Auf fast allen Kahlstellen hat eine Sortierung stattgefunden, die zur Bildung sekundärer Strukturböden geführt hat (Abb. 2 und 4). Die Kahlstellen als Ganzes aber scheinen nicht durch Sortierung entstanden zu sein, was ihre Klassifikation zu nonsorted circles rechtfertigt.

Der Versuch, die in Westspitzbergen gemachten Beobachtungen in diesem Gebiet zu bestätigen, erwies sich als nicht allzu positiv. So konnte beispielsweise bei keiner Aufgrabung eine seitliche Materialverlagerung nachgewiesen werden, wobei allerdings zu sagen ist, daß in dem dunklen Material des Bündnerschiefers Boden- und Horizontbildungen nur sehr schwer zu erkennen sind; aber auch Laboruntersuchungen in dieser Richtung verliefen negativ.

Nachfolgend einige Analysenresultate, die an einer Grabung (Fig. 3) gewonnen wurden:

Von der Korngröße her sind mit Ausnahme der Probe aus dem $\mathrm{Ah}_{\mathrm{h}}$-Horizont praktisch keine Unterschiede in der Zusammensetzung der Feinerde festzustellen (49-54\% Sand, $38-40 \%$ Schluff, $8-11 \%$ Ton). Der organische Kohlenstoffgehalt zeigt keine unnatürliche Verteilung, bemerkenswert sind aber doch die relativ hohen Werte von $0,5 \%$ in den untersten Bereichen. Diese Werte müssen aber nicht notwendigerweise nur auf organische Substanzen zurückzuführen sein, auch mineralische Substanzen in oxidierbarer Form, beispielsweise $\mathrm{Fe}^{2+}$, können zu ähnlichen Resultaten führen. Auch die pH-Werte weisen keine interpretierbaren Abweichungen von einer normalen Verteilung auf (nicht ganz verständlich erscheint aber der leichte 


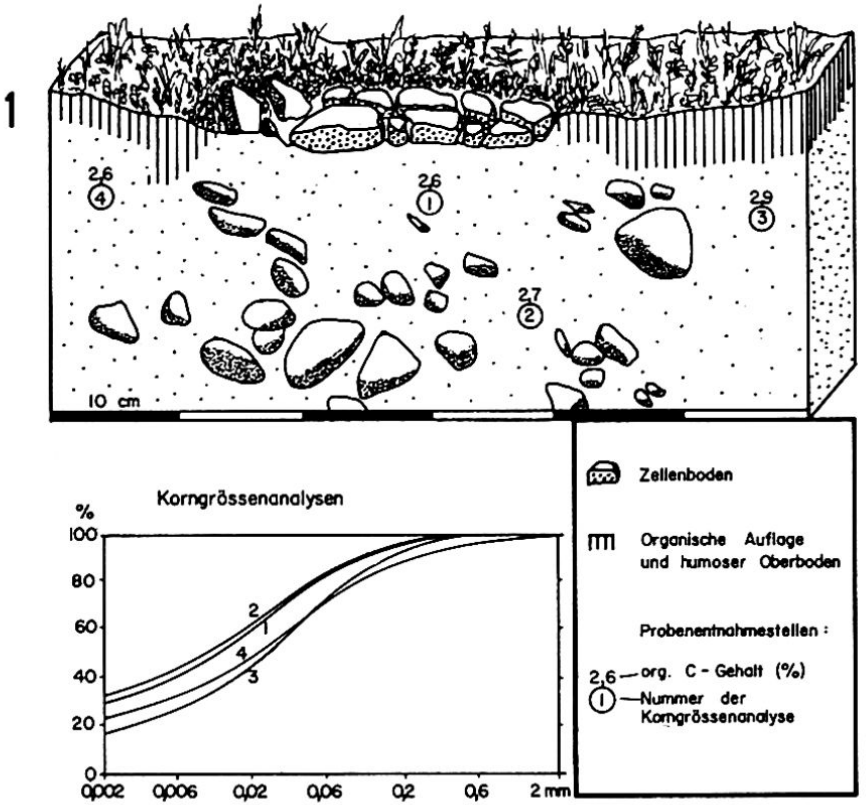

Fig. 1 Aufschlußskizze mit Analysenresultaten des in Abb. 1 fotographierten nonsorted circles (Erläuterungen im Text). Westspitzbergen

Fig. 2 Vertikalschnitt durch einen nonsorted circle mit Analysenresultaten (Erläuterungen im Text). Westspitzbergen

Fig. 3 Vertikalschnitt durch einen nonsorted circle mit Analysenresultaten (Erläuterungen im Text).

Col de l'Iséran
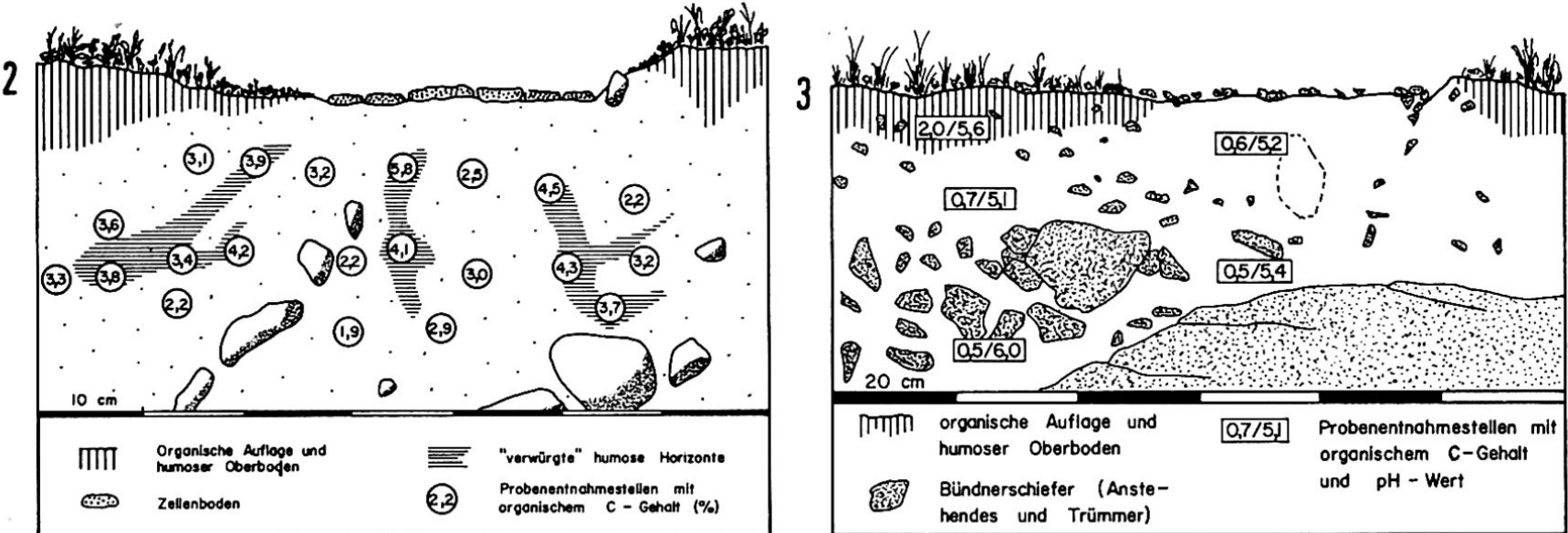


\section{Westspitzbergen}

Kahlstellen relativ klein und einheitlich ausgebildet

Oberfläche meist texturiert

(sekundärer Zellenboden)

Permafrostoberfläche in

60 bis $80 \mathrm{~cm}$ Tiefe

Hebungsvorgänge mit vertikaler

Materialverlagerung nachgewiesen

Horizontale Materialverlagerungen ziemlich sicher nachgewiesen

\section{Col de l'Iséran}

Kahlstellen relativ groß und Formen variabel

Oberfläche meist strukturiert

(sekundärer Steinnetzboden)

Permafrost nicht nachgewiesen, aber oft

Anstehendes in geringer Tiefe

Hebungsvorgänge mit vertikaler

Materialverlagerung nachgewiesen

Horizontale Materialverlagerungen

nicht nachgewiesen
pH-Anstieg im Ah-Horizont). Was nun das Skelettmaterial anbetrifft, so zeigt sich direkt unter der Kahlstelle ein deutlich niedrigerer Gehalt als daneben, also eine Art Feinerdekessel. Dies ist mit Sicherheit auf das Ausfrieren von Skelettmaterial zurückzuführen, welches an der Oberfläche das schon erwähnte Steinnetzwerk bildet. Eine nach oben gerichtete Bewegungskomponente kommt auch in einem vertikal stehenden Stein zum Ausdruck, der beim weiteren Aufgraben unter der Kahlstelle gefunden wurde (in Fig. 3 gestrichelt eingezeichnet).

Alle auf dem Col de l'Iséran gemachten Beobachtungen deuten auf einen engen genetischen Zusammenhang mit Strukturböden hin. Nicht ganz klar ist aber das Fehlen von Großformen von Strukturböden, die vom Material her auf dieser Höhe eigentlich $\mathrm{zu}$ erwarten sind, doch gibt möglicherweise die an dieser Stelle hoch hinaufreichende Vegetation den Hinweis, daß Großformen von Strukturböden erst in noch höheren Lagen zu erwarten sind (vgl. dazu FURRER und FITZE, 1971). Die in der Arktis nachgewiesenen partiellen seitlichen Materialverlagerungen konnten in diesem Untersuchungsgebiet nicht beobachtet werden, doch müßten für eine endgültige Abklärung noch bedeutend mehr Untersuchungen gemacht werden.

\section{Zusammenfassung}

Ein Vergleich zwischen nonsorted circles auf Westspitzbergen und am Col de l'Iséran ist in der obigen Zusammenstellung gegeben:

Es konnte eindeutig gezeigt werden, daß sowohl in der Arktis als auch in den Alpen vertikale Materialverlagerungen und damit verbunden Sortierungsvorgänge an der Genese von nonsorted circles mitbeteiligt sind. Der Begriff «nonsorted circle» ist also im Grunde genommen nach diesen Ausführungen nicht korrekt. Wichtige Indizien für diese Art von Materialverlagerung sind die Steilstellung von größeren Steinen und Humuszonen unter der Kahlstelle, die Bildung von «Feinerdekesseln» und (was nicht als Gegensatz aufgefaßt werden darf) die Konzentration von größeren Steinen im Untergrund der Formen. Zudem ist oft eine gewisse Sortierung an der Oberfläche der Formen direkt zu sehen.

Materialpressungen im Untergrund und daraus resultierende partielle seitliche Materialverlagerungen konnten nur in Spitzbergen mit einiger Sicherheit nachgewiesen werden

Aus diesen Gründen sowie auch aus der doch verschiedenen Ausprägung der nonsorted circles in der Arktis und in den Alpen kann geschlossen werden, daß die Genese in den beiden Fällen nicht ganz identisch ist. 
Ich danke der Stiftung für wissenschaftliche Forschung an der Universität Zürich für die finanzielle Unterstützung der Feldarbeit.

\section{Anhang}

Die Untersuchungen im Labor wurden nach folgenden Methoden durchgeführt:

Organische Kohlenstoffbestimmung

Oxidation des Kohlenstoffs mit $\mathrm{K}_{2} \mathrm{Cr}_{2} \mathrm{O}_{7}$ in schwefelsaurer Lösung nach der Methode von Walkley-Black (in JACKSON, 1958). Gegenüber der trockenen Veraschung werden nur 75 bis $80 \%$ des organischen Kohlenstoffs erfaßt.

\section{Korngrößenanalyse des Feinerdematerials}

Naßsiebung (20 Minuten) für die Sandfraktion (2 bis $0,06 \mathrm{~mm}$ ), Aräometeranalyse für Schluff- und Tonfraktion

pH-Wert

Messung mit der Glaselektrode in ln $\mathrm{KCl}$-Lösung

\section{Literatur:}

DEBELMAS, J.: Guides Géologiques Régionaux: Alpes (Savoie et Dauphiné); Paris, 1970

FURRER, G. und FITZE, P.: Die Höhenlage von Solifluktionsformen und der Schneegrenze in Graubünden; Geogr. Helv. 26/3, 1971
GRAF, K.: Beiträge zur Solifluktion in den Bündner Alpen (Schweiz) und in den Anden Perus und Boliviens; Diss. Univ. Zürich, 1971

GRIPP, K.: Beiträge zur Geologie von Spitzbergen; Abh. des Naturw. Ver. zu Hamburg, XXI/3, 1927

HOPKINS, D. M. und SIGAFOOS, R. S.: Frost action and vegetation patterns on Seward Peninsula, Alaska; Geological survey bulletin, 974-C, Washington, 1951

HOPKINS, D. M. und SIGAFOOS, R. S.: Role of frost thrusting in the formation of tussocks; Am. Journ. of Science, 252,1954

JACKSON, M. L.: Soil chemical analysis; London, 1958 LUNDQVIST, J.: Patterned ground and related frost phenomena in Sweden; Stockholm, 1962

MAJOR, H. und NAGY, J.: Geology of the Adventdalen map area; Norsk Polarinstitut Skrifter, 138, 1972

SEMMEL, A.: Verwitterungs- und Abtragungserscheinungen in rezenten Periglazialgebieten (Lappland und Spitzbergen); Würzb. Geogr. Arb., 26, 1969

WASHBURN, A. L.: Classification of patterned ground and review of suggested origins; Geol. Soc. of America, 67,1956

WASHBURN, A. L.: Weathering, frost action, and patterned ground in the Mesters Vig District, Northeast Greenland; Meddelelser om Grönland, 176/4, 1969

WASHBURN, A. L.: Periglacial processes and environments; London, 1973 